dehydrated (five had had gastric surgery, one was taking $\mathrm{H}_{2}$ receptor blockers) while the others generally had a milder illness and were often recovering by the time the results of blood cultures were available.

\section{Comment}

The American studies were based on data from patients in the community as well as in hospitals ${ }^{12}$ and thus are not strictly comparable with our study, which found a higher rate of bacteraemia among patients with salmonellosis in hospital. Two factors influenced this rate: the serotype and age. The rates varied widely with serotype, with $S$ dublin, $S$ infantis, $S$ virchow, $S$ panama, and $S$ newport being the five most invasive strains; the invasiveness of $S$ dublin and $S$ virchow has been reported before. ${ }^{2-4}$ The vulnerability of elderly patients to bacteraemia and to severe dehydration and often fatal illness was highlighted. Associated debilitating states and the decline in cell mediated immunity with age are probably important in the pathogenesis of bacteraemia in the elderly. In the absence of comparable clinical data on patients with non-bacteraemic salmonellosis we cannot comment on whether patients with bacteraemia have a more severe illness. The mortality statistics, however, suggest that this may be so.

Antibiotics are not generally advocated in salmonella gastroenteritis unless there is associated septicaemia, but this is difficult to determine clinically during the early stages of the illness, when the antibiotics will be most successful. Delaying treatment until results of blood culture are available may result in dehydration related to septicaemia and renal failure. Fever and leucocytosis are unreliable indicators of bacteraemia. Perhaps a lower threshold is needed for early use of antibiotics in elderly patients with severe diarrhoea.

1 Cherubin CE, Neu HC, Imperato PJ, Harvey RP, Betten N. Septicaemia with non-typhoid salmonella. Medicine (Baltimore) 1974;53:365-76.

2 Blaser MJ, Feldman RA. Salmonella bacteraemia: reports to the Centres for Disease Control, 1968-1979. I Infect Dis 1981;143:743-6.

3 Mani V, Brennand J, Mandal BK. Invasive illness with Salmonella virchow infection. BrMed f 1974;ii: 143-4.

4 Todd WT, Murdoch JM. Salmonella virchow: a cause of significant blood stream invasion. Scott Med f 1983;28:176-8.

(Accepted 1 September 1988)
Department of Radiology and University Department of Medicine, General Infirmary, Leeds LS1 3EX C E Williams, FRCR, senior registrar

B M Carey, FRCR, senior registrar

A J Birtwell, MRCP, lecturer J K Wales, FRCP, senior lecturer

P G Wiles, MRCP, lecturer in medicine

Correspondence to: Dr Wiles.

\section{Metatarsal periosteal reactions: a common non-specific finding in radiographs of the diabetic foot}

\author{
C E Williams, B M Carey, A J Birtwell, \\ J K Wales, P G Wiles
}

Periosteal reaction, when evident in a radiograph of a diabetic foot, is generally considered to indicate osteomyelitis. We have, however, observed metatarsal periosteal reactions in radiographs of the feet of diabetic patients without other evidence of osteomyelitis whose foot problems have subsequently resolved with local treatments alone-that is, without systematic antibiotics. We reviewed a large representative group of diabetic patients and determined the prevalence of metatarsal periosteal reactions.

\section{Patients, methods, and results}

Radiographs of the feet were obtained for the first 195 diabetic patients ( 50 with type I diabetes and 145 with type II; median age 65 (range 19-87); 99 men and 96 women) attending a foot screening clinic. All radiographs were reviewed independently by two radiologists without knowledge of clinical findings. Any radiographs over which they disagreed were not included in the results. A control group, individually matched for age and sex, was compiled from patients attending the casualty department who required foot radiographs for what proved to be unimportant soft tissue injuries. Films were assessed for the presence of periosteal reaction, subluxation, fractures, and vascular calcification. Statistical analyses were performed by McNemar's test for paired samples and $\chi^{2}$ and MannWhitney $\mathrm{U}$ tests for unpaired samples.

Periosteal reactions were found in 42 diabetic patients ( 21 men) but only seven controls (four men), $(p<0 \cdot 001)$. Disagreement over the radiological interpretation excluded a further five patients and one control from the study. The periosteal reaction was on both sides of the metatarsal shaft in 11 of the 21 women and six of the 21 men and was generally thick and without demarcation from the cortex (figure). Second, third, and fourth metatarsals were most commonly

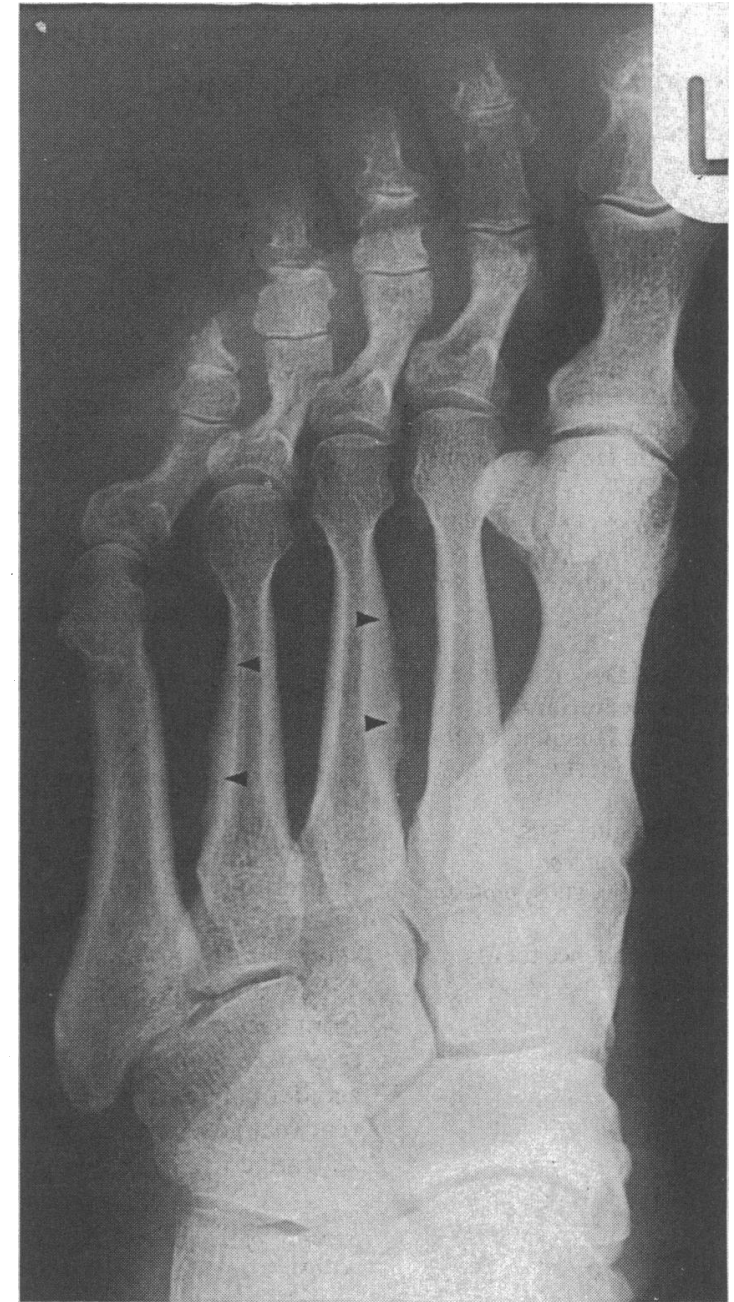

Radiograph of left foot showing thick periosteal reactions along shafts of third and fourth metatarsals

affected and more often in combination than individually. None of the patients had trophic ulcers or clinical evidence of infection or fracture. The presence of periosteal reaction was not associated with other radiological abnormalities, age, duration of diabetes (perhaps because of the preponderance of patients with type II diabetes), or clinical neuropathy (absence of 
ankle jerks and vibration sense at the first metatarsophalangeal joint). Vibration perception threshold at the great toe was measured in 101 of the diabetic patients (23 with periosteal reaction) and was significantly higher in those with periosteal reactions (median 30.5 v 20 units, $\mathrm{p}<0.05$ ).

\section{Comment}

Periosteal reaction has been observed in diabetes ${ }^{1}$ and in association with trophic ulcers ${ }^{2}$ and neuropathic joint disease. ${ }^{3}$ It was not, however, mentioned in a review of foot radiographs in 162 diabetic patients with gangrene. ${ }^{4}$ Formation of new bone at the metatarsal periosteum, perhaps resulting from previous fracture, has been reported in diabetes, ${ }^{5}$ but its nature seems quite different from the pattern of periosteal changes described here.

Periosteal reaction in an ulcerated foot is generally believed to indicate infection in or around the affected bone. We found metatarsal periosteal reactions in radiographs of asymptomatic diabetic feet without evidence of infection, fracture, or ulceration. The cause is unknown, but the association with raised vibration perception threshold suggests a common (perhaps vascular) aetiology. Osteomyelitis can be particularly severe in the diabetic foot, and infection should always be considered when a localised periosteal reaction is present. Our results, however, suggest that metatarsal periosteal reactions are common among diabetic patients and do not of themselves indicate osteomyelitis.

We thank Mrs J Papuga for typing the manuscript.

1 Staple TW. Radiography of the diabetic foot. In: Levin ME, O'Neal LW, eds. The diabetic foot. St Louis: C V Mosby, 1983:201-31.

Rubin LM. Periostitis associated with diabetic ulcer. $f$ Am Podiatr Med Assoc 1969;59:154-5.

3 Verlickov L, Djankov L. Periostreaktionen bei diabetischen Osteoarthropathien. Radiol Diagn (Berl) 1971;12:107-13.

4 Lither F, Heitala SO, Steen L. Skeletal lesions and arterial calcifications of the feet of diabetics. Acta Med Scand [Suppl] 1984;687:47-54.

5 Newman JH. Non-infective disease of the diabetic foot. In: Connor H, Boulton AJM, Ward JD, eds. The foot in diabetes. Chichester: Wiley, 1987:81-90.

(Accepted 5 August 1988)

\title{
Incidence and consequences of ejection in motor vehicle accidents
}

\author{
G F McCoy, R A Johnstone, \\ I W Nelson, J Kenwright, R B Duthie
}

\begin{abstract}
Oxford Road Accident
Group, Nuffield

Department of

Orthopaedic Surgery, John

Radcliffe Hospital, Oxford OX3 9DU

G F McCoy, FRCs, research fellow

R A Johnstone, BA, research assistant
\end{abstract}

\section{Nuffield Department of} Orthopaedic Surgery, John Radcliffe Hospital, Oxford I W Nelson, FRCS, clinical lecturer

J Kenwright, FRCS, consultant surgeon

R B Duthie, FRCs, professor

Correspondence to: $\mathrm{Ms}$ Johnstone.
A widespread misapprehension exists that people thrown clear from a vehicle in an accident have a lower risk of serious injury than those remaining inside, though published reports generally contradict this view. ${ }^{12}$ There are, however, few reports on the incidence of ejection in restrained and unrestrained vehicle occupants and the consequences in terms of severity of injury. We studied people who were ejected completely from their vehicles and compared their severity of injury with that of the large number of people who were not ejected.

\section{Patients, methods and results}

From January 1983 to January 1985 information was collected prospectively by this group on all patients injured in road traffic accidents in the catchment area of this hospital. Injuries were coded by region and scored with the abbreviated injury scale. ${ }^{3}$ Altogether 1550 vehicle occupants were injured (871 drivers, 375 front seat passengers, 304 rear seat passengers). Forty five were completely ejected from the vehicle in the accident ( 11 drivers, nine front seat passengers, and 25 rear seat passengers; 27 male and 18 female; mean age 25 (range 1 to 51 )). The incidence of ejection was $2.9 \%$

Severity of injury and deaths in ejected and non-ejected vehicle occupants

\begin{tabular}{lcc}
\hline $\begin{array}{l}\text { Maximum abbreviated } \\
\text { injury scale }\end{array}$ & No (\%) not ejected & No (\%) ejected \\
\hline 1 and 2 & $1179(78 \cdot 3)$ & $22(48 \cdot 9)$ \\
3 & $231(15 \cdot 4)$ & $9(20 \cdot 0)$ \\
4 and 5 & $35(2 \cdot 3)$ & $10(22 \cdot 2) \dagger$ \\
Deaths & $60 \ddagger(4 \cdot 0)$ & $4(8 \cdot 9)$ \\
\hline \multicolumn{1}{c}{ Total } & 1505 & 45 \\
\hline
\end{tabular}

$\star 1$ And $2=$ minor $/$ moderate; 4 and $5=$ severe/life threatening.

$+\chi^{2}=51 \cdot 1 ; \mathrm{df}=3 ; \mathrm{p}<0 \cdot 0001$

$\neq$ Three people died from natural causes while driving. overall. It was lower for drivers $(1 \cdot 2 \%)$ and front seat passengers $(2 \cdot 4 \%)$ but significantly higher for rear seat passengers $(8 \cdot 2 \%)\left(\chi^{2}=42 \cdot 5 ; \mathrm{df}=1 ; \mathrm{p}=<0.001\right)$.

The table shows the consequences of ejection in terms of increased severity of injury and death. Being ejected was associated with a significantly greater incidence of severe or critical injury $\left(\chi^{2}=51 \cdot 1\right.$, $\mathrm{p}<0.0001)$ and a higher incidence of fatal injuries. Deaths were due to severe head injury (two), laceration of the aorta (one), and multiple fractures of long bones with severe contusion of the lung (one). The most important sites of injury in severely injured patients were the abdomen (five patients), chest (two), head (two), and spine (one).

None of the ejected rear seat passengers were wearing a seat belt. Of the 834 occupants known to be wearing seat belts, only five were ejected (two front seat passengers and three drivers). We did not know whether the remaining 218 drivers and front seat passengers were wearing seat belts; six of them were ejected. Two small children travelling on the laps of front seat passengers were ejected. Ejection occurred most commonly in accidents at high speeds and those in which the vehicle rolled over.

\section{Comment}

Our data show that rear seat passengers have a higher risk of ejection. None of the ejected rear seat passengers were wearing seat belts. In the front seats five of the 20 ejected occupants (drivers and passengers) were known to be wearing seat belts. The rate of ejection was $0.6 \%$ for those known to be wearing seat belts and $7 \cdot 3 \%$ for those known not to be wearing them. Clearly seat belts safeguard against ejection, and this is particularly relevant in the rear seat, an observation in keeping with the findings of Hobbs. ${ }^{5}$

When ejection occurred the incidence of fatal injury was over twice that for the non-ejected group, a finding supported by other studies. ${ }^{14}$ The difference between ejected and non-ejected people was most apparent in the severely or critically injured group, ejected people being far more likely to sustain such injuries.

In conclusion, ejection from a vehicle in an accident results in a much higher rate of serious injury and death than would otherwise occur. The incidence of ejection depends heavily on seating position and is reduced by the proper use of restraints. Fitting and using restraints in the rear seat, where the risk of ejection is greater, 